\title{
Which Are The Most Relevant Comorbidities
}

\section{In COPD?}

Miguel Divo, MD ${ }^{1}$ and Carlos Cabrera, MD²

${ }^{1}$ Pulmonary and Critical Care Division, Brigham and Women's Hospital, Harvard Medical School, Boston, Massachusetts, USA; ${ }^{2}$ Pulmonary Department, Hospital Universitario de Gran Canaria Dr Negrin, Las Palmas de Gran Canarias, Spain

\section{ABSTRACT}

The world's population is aging. A person who does not die of acute illnesses will likely develop more than one non-communicable disease, a phenomenon known as multimorbidity.

COPD is the third leading cause of death worldwide and is defined by airflow limitation, but its clinical manifestations are variable and influenced by comorbidities. Comorbidity burden can be measured by the number of coexisting diseases, by their prevalence, and by the impact on outcomes.

There are limitations when traditional epidemiology methods are applied to study the complex interactions amongst many diseases as the relevance of some diseases may mistakenly omitted. We present in this review the results from the most influential COPD comorbidity studies, highlighting those that have provided the most relevant information, and then we present a novel perspective derived from network analysis, which helps interpret the complex interplay among comorbidities. (BRN Rev. 2016;2:215-28)

Corresponding author: Miguel Divo, mdivo@partners.org

Key words: Cluster. Comorbidity. Chronic obstructive pulmonary disease. Network. Outcomes.

\section{Correspondence to:}

Miguel Divo, MD

Pulmonary and Critical Division

Brigham and Women's Hospital

75 Francis Street

Received in original form: 05-08-16

Boston, Massachusetts 02115, USA

E-mail: mdivo@partners.org

Accepted in final form: 25-08-16

DOI: 10.23866/BRNRev:2016-M0028 


\section{INTRODUCTION}

The World's population is growing older and chronic diseases are more prevalent. Advances in the treatment and control of communicable diseases have led to a steady increase in life expectancy worldwide. Currently, life expectancy is 78 years in the developed world and 68 years in the developing world, whereas in 1950, life expectancy at birth was 65 years in high-income countries and 42 years in lowand middle-income countries ${ }^{1}$. With the aging of the world's population, non-communicable or chronic diseases are the predominant challenge for medicine. The success of the methods applied to the control and treatment of communicable diseases is not necessarily easy to extrapolate to the control and treatment of chronic diseases, and therefore limits the application of conventional approaches to this new health reality.

Communicable diseases have a relatively short time interval from exposure to disease manifestation, allowing scientist to reproduce experiments and see results in relatively short periods of time. Chronic diseases develop slowly and manifest at older age, inducing a perception of less urgency in the society. Second, communicable diseases result from a single or small number of interactions with infectious agents or vectors (air, food, or vector borne). This characteristic allows a simplified mapping of disease mechanisms and therefore the identification of a therapeutic target. For example, in the recent Ebola outbreak, identification of the virus' life cycle and ribonucleic acid (RNA) code enabled scientists to control the spread (despite a locally limited health system) and develop two potential vaccine candidates in a period of two years $^{2-4}$. Third, and most significantly, multiple chronic diseases co-occur in the same individual, a phenomenon known as "multimorbidity" and is a reality of today's aging population $^{5,6}$. Aging with multiple coexisting chronic diseases has caught the present healthcare system unprepared because the reductionist "silo" approach that was successful in preventing and treating communicable diseases has not borne the same results for chronic multimorbidity. Unfortunately, the reductionist approach is the basis of the current structure of medical education, healthcare delivery, drug discovery, and practice guidelines. Therefore, a different approach is needed and it will be discussed in this review.

The top-ranked chronic diseases in the world include heart attack, stroke, chronic obstructive pulmonary disease (COPD), diabetes, cancer, and depression? ${ }^{7}$. Since 1990, COPD has climbed from fifth to third place among the leading causes of death worldwide ${ }^{7}$ (Fig. 1) and was responsible for three million deaths in 2010 (6\% of all deaths).

While it is important to rank diseases by their frequency, the reality is that those chronic diseases can co-occur in the same individual and this phenomenon seems not to occur by chance alone ${ }^{8}$. In fact, for COPD, more than $80 \%$ of patients have concomitant heart disease, cancer, osteoporosis, and depression, among others. This was demonstrated in one of the seminal COPD trials, the Towards a Revolution in COPD Health (TORCH) trial, which aimed to measure mortality as an outcome. In this study, researchers carefully tracked specific causes of death of 6,000 patients over three years. Only $35 \%$ of the 875 deaths were due to primary respiratory causes, while $27 \%$ 
Global

1990 rank

Both sexes, All ages, Deaths per 100,000

\begin{tabular}{|l}
\hline 1 Ischemic heart disease \\
\hline 2 Cerebrovascular disease \\
\hline 3 Lower respiratory infect \\
4 Diarrheal diseases \\
\hline 5 COPD \\
6 Tuberculosis \\
7 Neonatal preterm birth \\
8 Road injuries \\
9 Lung cancer \\
10 Malaria \\
\hline
\end{tabular}

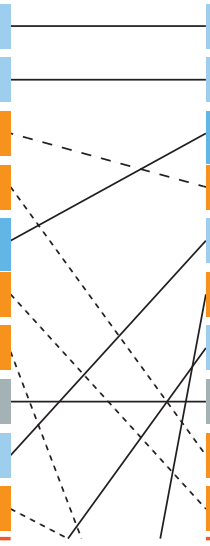

2010 rank 1 Ischemic heart disease 2 Cerebrovascular disease

\section{COPD}

4 Lower respiratory infect

5 Lung cancer

6 HIV/AIDS

7 Alzheimer disease

8 Road injuries

9 Diarrheal diseases

10 Tuberculosis

Figure 1. Top 10 causes of death worldwide comparing 1990 to 2010. Data include all ages, both genders. Table was purposely truncated to show the top 10 causes. Institute for Health Metrics and Evaluation (IHME). GBD Compare. Seattle, WA: IHME, University of Washington, 2015. Available at: http://vizhub.healthdata.org/gbd-compare [Accessed 03.18.2016].

died from heart problems, and $21 \%$ due to cancer ${ }^{9}$. Of these cancers, close to $80 \%$ were lung cancers.

For COPD, the importance of considering comorbidities as part of its core management has evolved from simply acknowledging their existence, to recognizing their impact on important outcomes and, more recently, to describing how certain comorbidities cluster preferentially around particular COPD phenotypes $^{1,10-16}$.

\section{DEFINITIONS}

In table 1 we have listed important concepts used throughout this review to facilitate its comprehension.

\section{DETECTING COMORBIDITIES}

Comorbidity ascertainment is a relevant but overlooked aspect in the methodology of comorbidity studies. The three most common methods to ascertain comorbidities are by: (i) direct questioning or anamnesis, (ii) confirmation with specific diagnostic test, and (iii) mining administrative databases. We will briefly review each methodology, comparing their strengths and weaknesses.

First, anamnesis or history taking has been for centuries the cornerstone of medical information. This methodology provides the chance to capture a wide spectrum of comorbidities and can be enhanced by combining open-ended history taking with questionnaire-directed examination. To decrease recall bias, each 
TABLE 1.

Key definitions and concepts

Comorbidity and multimorbidity Comorbidity is defined as the presence of one or more diseases co-occurring with a disease of interest or index disease. In contrast, multimorbidity does not assume a hierarchy of diseases (Fig. 2). The indexed disease does not necessary represent the dominant or the cause of the other chronic disorders, but rather helps to determine a reference that is useful for a case-control study.

Communicable and non-communicable diseases

Communicable or infectious diseases are those caused by pathogenic microorganisms the diseases can be spread, directly or indirectly, from one person to another. On the other hand, non-communicable diseases are not passed from person to person. They are of long duration and generally slow progression; non-communicable diseases are also known as chronic diseases; however, some infectious disease may become chronic (hepatitis C for example).

Cluster analysis

This is the task of grouping a set of objects in such a way that objects in the same group (called a cluster) are more similar (in one sense or another) to each other than to those in other groups.

Network analysis

This is a useful way to conceptualize and present graphically a complex system, where the different elements of the system are represented as nodes and their structural and/or functional relationship as edges (or links) $)^{28,29}$.

Modules

This is a dense sub-graph on the network that often represents a set of nodes that have a joint role ${ }^{40}$.

Hubs Nodes in a networks that possess the highest number of links or connections ${ }^{40}$.

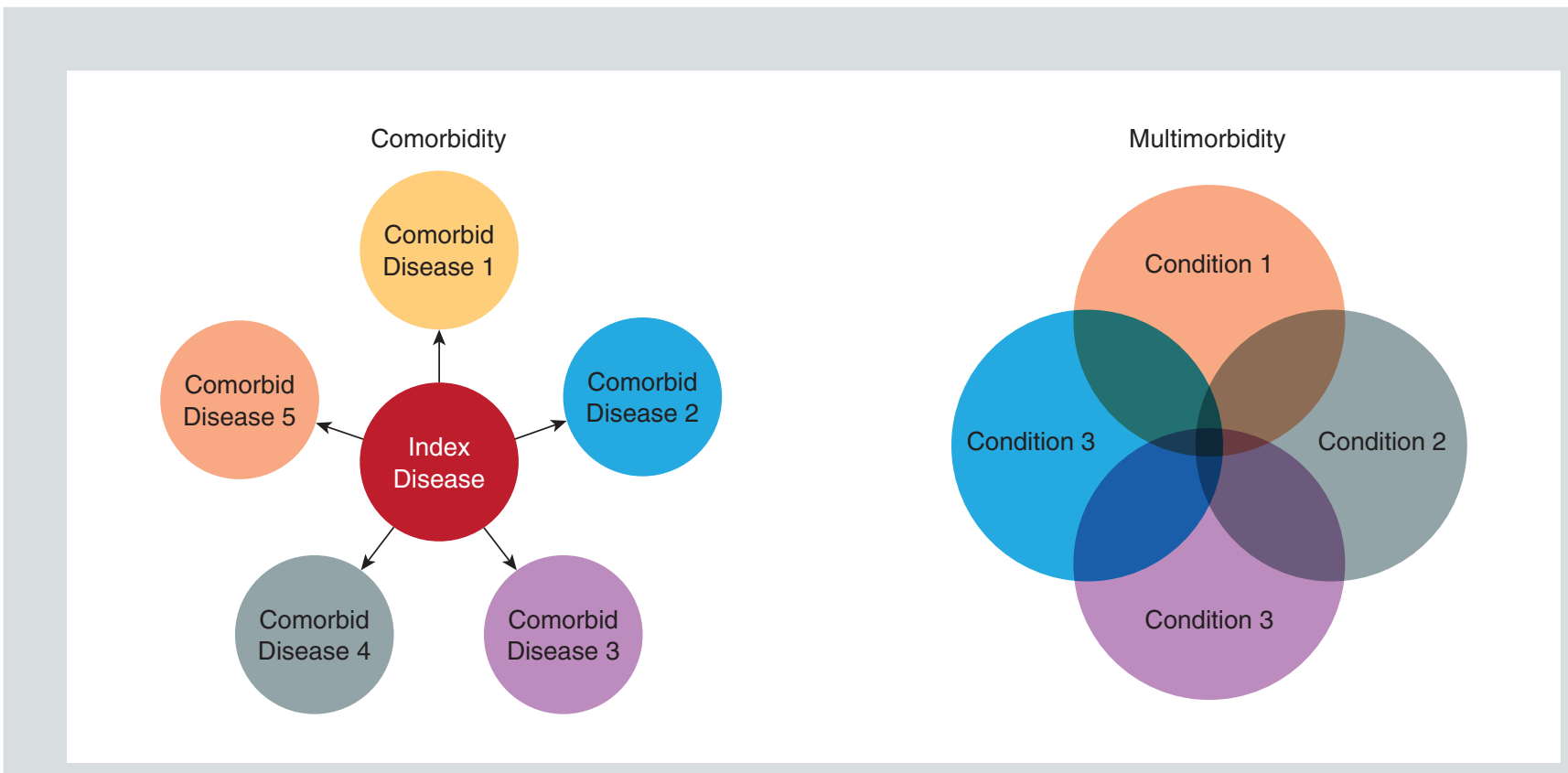

Figure 2. Graphical representation comparing the concepts of comorbidity and multimorbidity.

diagnosis can be confirmed (or the list expanded) by reviewing other laboratory, radiologic, and pulmonary function test results in the patient's chart.
Second, direct measurements by means of confirmatory diagnostic testing. This method provides a more objective ascertainment and accurate estimate of comorbidity prevalence, 
avoiding the pitfall of recall bias, under-diagnoses, or missing subclinical comorbidity. However, the objectivity of these methods is counterbalanced by the limitation of how many diagnostics test can epidemiologic studies afford, the applicability to clinical practice, and the risk implicit in some tests (i.e. radiation) resulting in the screening for a handful of comorbidities and therefore introducing selection bias.

The third method is the examination of databases. This method has the advantage of scaling studies to include thousands (or millions) of patients. However, in many cases there is no way to confirm the diagnosis, and in the USA many databases were conceived for administrative-billing claim purposes and not necessarily clinically driven.

When comparing the three methods, the first will provide a wide range of different comorbidities with a number of participants ranging from hundreds to a few thousands. For the second method, the number of different comorbidities are narrower, with fewer patients but more accurate prevalence estimates, especially in those diseases that are diagnosed by imaging (Fig. 3). For the last method, there is a wide range of comorbidities and number of patients, but it is difficult to discern errors of misclassification or missing data.

\section{WHICH COMORBIDITIES?}

To better appreciate the importance of comorbidities, we should understand the different dimensions of comorbidities burden:
- By the cumulative number of different diseases one individual has and their prevalence in the population;

- By the impact on patient's centred outcomes: mortality, response to treatment, hospitalizations, and quality of life;

- How comorbidities may cluster according to particular COPD phenotypes.

\section{Number of comorbidities and prevalence}

Multimorbidity is a known phenomenon of aging ${ }^{5}$; however, having COPD, the odds of carrying multiple comorbidities is 1.5 -fold higher compared to a matched non-COPD control group $^{17-21}$ (Fig. 4).

Not only is the number of cumulative diseases higher in COPD patients, but also the prevalence of many co-occurring diseases is higher compared to controls, as is illustrated in table 2. In that table, we organized the results from several COPD comorbidity studies that used multiple rather than one comorbidity in their analysis, and compare them by the method of measuring the comorbidity's prevalence, the study setting, and the number of patients included. Some facts stand out: (i) the prevalence of comorbidities is higher in patients with COPD compared to controls; (ii) there is variability in the prevalence estimate between studies, and this seems to relate to the setting and methodology used in the study; (iii) cardiovascular, locomotive, psychiatric, and metabolic diseases are the most prevalent comorbidities in the majority of studies ${ }^{22-25}$. 


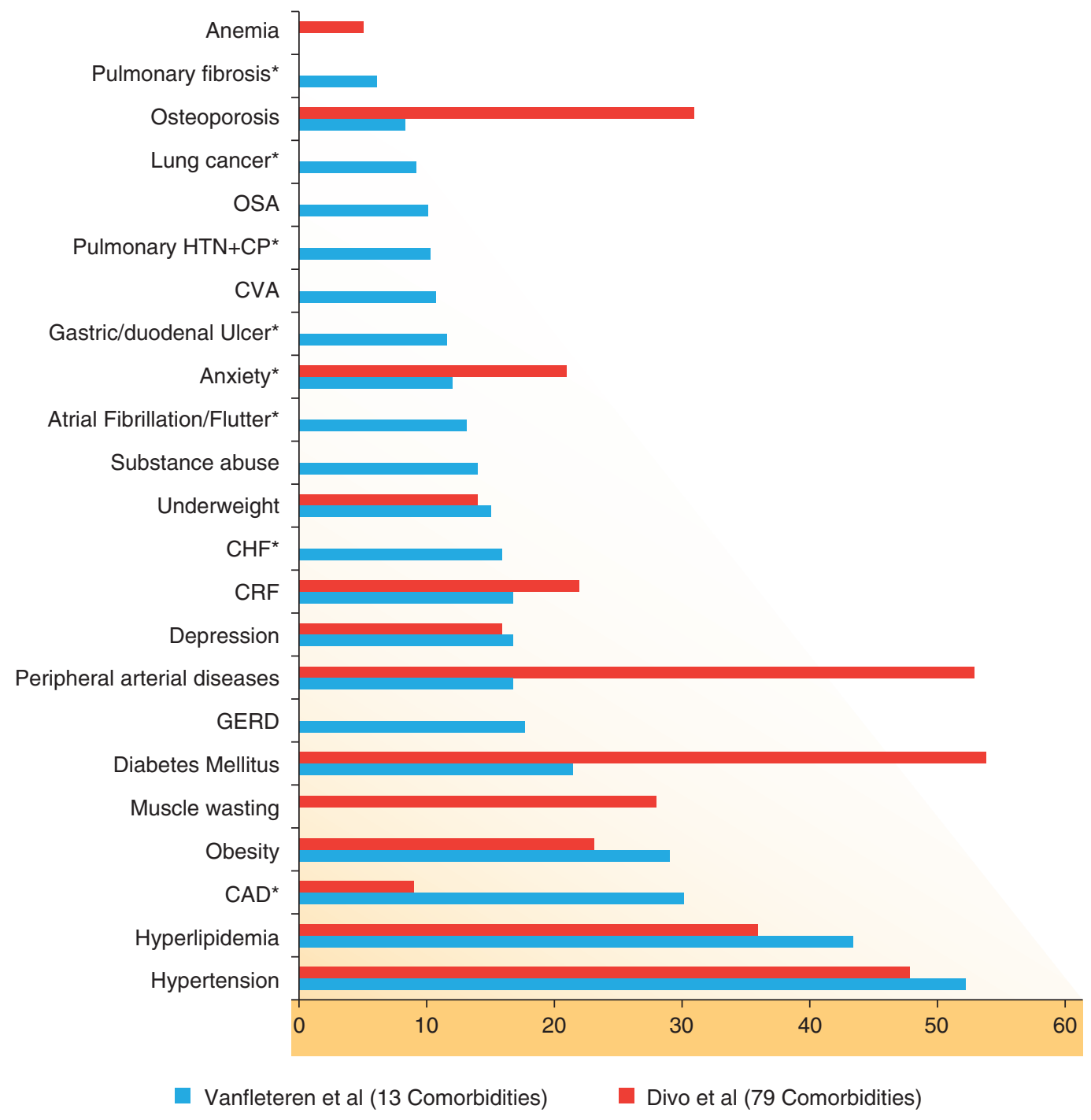

Figure 3. Prevalence comparison between two COPD comorbidities studies using two different methods to ascertain comorbidities prevalence. In red bars are the values measured by direct measurement of 13 diseases in a cohort of 213 patients ${ }^{13}$ and in blue by history taking and chart review in 1,659 patients accounting for 79 different comorbidities ${ }^{35}$. Note a wider difference in prevalence in those diseases that require specialized testing.

CAD: coronary artery disease; CHF: congestive heart failure; CP: cerebral perfusion; CRF: chronic respiratory failure; CVA: cerebrovascular accident; GERD: gastro-oesophageal reflux disease; HTN: hypertension; OSA: obstructive sleep apnoea.

\section{Impact on outcomes}

COPD is a disabling disease, affecting a patient's quality of life, and increasing the risk of hospitalizations and death. We learned from the Evaluation of COPD Longitudinally to Identify Predictive Surrogate Endpoints (ECLIPSE) study ${ }^{9}$ that the degree of airway obstruction alone cannot identify by itself patients at risk of developing those 


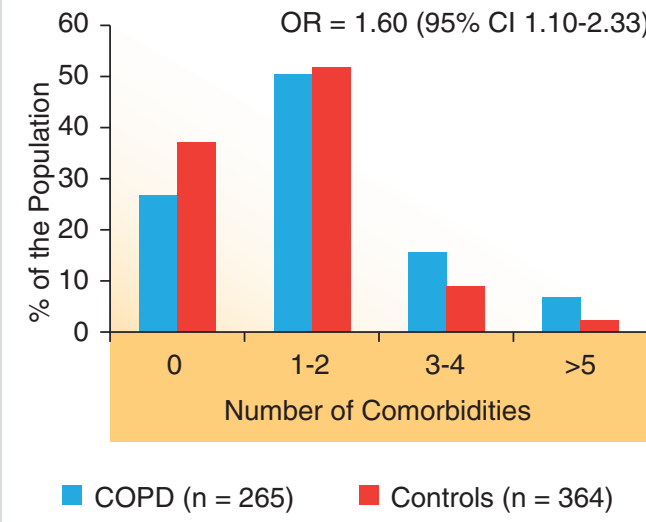

Figure 4. Cumulative number of comorbidities comparing patients with COPD and non-COPD matched controls (reproduced with permission from Van Mannen et al. ${ }^{17}$. J Clin Epidemiol. 2001;54:287-93).

complications. The development of multidimensional indices has changed the way we understand this disease and helped explain this variability, yet several studies have also demonstrated that comorbidities also add to this variability by impacting patient-centred outcomes. In table 2 we present the most influential COPD comorbidity studies, comparing important characteristic, such as population studied and number of participants, and the methodology used to ascertain comorbidities, and highlighted those comorbidities that have direct impact on outcomes (first column in bold): ischemic heart disease, congestive heart failure, cor pulmonale, lung cancer, diabetes (with end-organ damage), gastro-oesophageal reflux, peptic ulcer disease, cancers with emphasis on lung cancer, pulmonary fibrosis, degenerative joint disease, mental health (anxiety and depression), peripheral artery disease, chronic renal failure, atrial fibrillation, hyperlipidaemia, and hypertension.
However, important questions still remain: What is the significance of other comorbidities that may be present in patients with COPD and have no direct association with outcomes? Are they innocent bystanders?

\section{Diseases networks and comorbidity clusters}

The above questions are important if we want to understand the underlying mechanisms behind multimorbidity, and we have to recognize that the complexity of multiple disease interactions cannot be adequately assessed by standard regression analysis or be fitted into a linear equation. One alternative to address this problem is the use of clustering techniques, with the intent to differentiate subgroups of COPD patients with particular phenotypic and prognostic characteristics ${ }^{12-16,26,27}$, and expand to include comorbidities ${ }^{13-16}$. Despite the methodological differences between these studies, there is congruence in the identification of three to four COPD sub-groups. Some of the major clustering determinants are the degree of emphysema, airway obstruction, and the body mass index or fat-free mass. This type of analysis suggests that the response to tobacco exposure is expressed differently in susceptible individuals and the effect of such exposure goes well beyond the lungs.

One particular integrative approach is the use of network analysis. Networks are used to analyse other complex systems such as the World Wide Web, cell phone communications, and social networks. These complex systems are composed of numerous units (cell phones, computers, or friends) connected by links (text messages, phone calls, posts) and therefore 
TABLE 2. Summary of COPD comorbidities studies describing the setting, methodology of disease ascertainment, number of subjects, prevalence, and impact on important outcomes

\begin{tabular}{|c|c|c|c|c|c|c|c|c|c|c|c|c|c|c|c|}
\hline \multirow{3}{*}{$\begin{array}{l}\text { Disease } \\
\text { ascertainment }\end{array}$} & \multirow{2}{*}{ 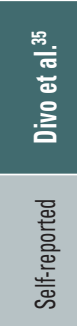 } & \multirow{2}{*}{ 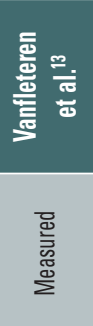 } & \multicolumn{2}{|c|}{ 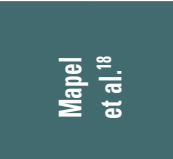 } & \multicolumn{2}{|c|}{ 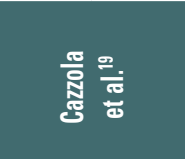 } & \multicolumn{2}{|c|}{ 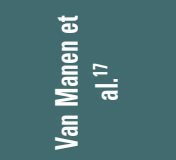 } & \multicolumn{2}{|c|}{ 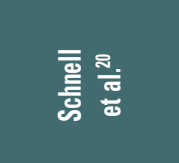 } & 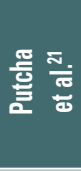 & 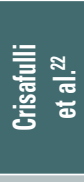 & 을 & \multirow[t]{2}{*}{ 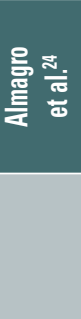 } & \multirow[t]{2}{*}{ 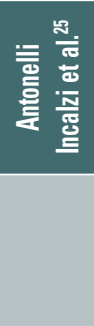 } \\
\hline & & & \multicolumn{2}{|c|}{ 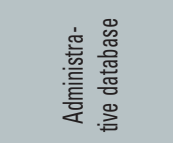 } & \multicolumn{2}{|c|}{ 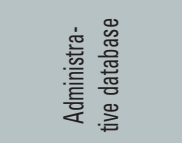 } & \multicolumn{2}{|c|}{ 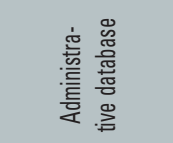 } & \multicolumn{2}{|c|}{ 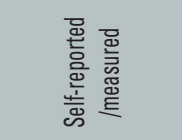 } & 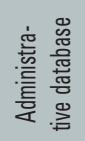 & 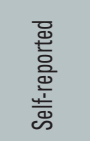 & 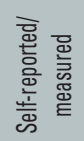 & & \\
\hline & COPD & COPD & COPD & $\begin{array}{l}\text { Con- } \\
\text { trols }\end{array}$ & COPD & Control & COPD & Control & COPD & Control & COPD & COPD & COPD & COPD & COPD \\
\hline n & 1,659 & 213 & 200 & 200 & 15,000 & 325,000 & 290 & 421 & 995 & 14,828 & 843 & 2,962 & 288 & 606 & 288 \\
\hline Outcome & 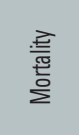 & & & & & & \multicolumn{2}{|c|}{ 욤 } & & & 용 & 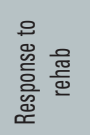 & 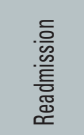 & 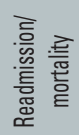 & 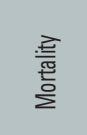 \\
\hline Setting & $\begin{array}{l}\text { Outpa- } \\
\text { tient }\end{array}$ & Acute & \multicolumn{2}{|c|}{ Outpatient } & \multicolumn{2}{|c|}{ Outpatient } & \multicolumn{2}{|c|}{ Outpatient } & \multicolumn{2}{|c|}{ Outpatient } & $\begin{array}{l}\text { Outpa- } \\
\text { tient }\end{array}$ & Acute & Acute & Acute & Acute \\
\hline \multicolumn{16}{|l|}{ Prevalence $(\%)$} \\
\hline Hypertension & 52 & 48 & 45 & 37 & & & $23^{*}$ & 21 & 60 & 52 & & $38^{*}$ & & 63 & \\
\hline Hyperlipidemia & 44 & 36 & & & & & & & 48 & 41 & & $9^{*}$ & 8 & & \\
\hline CAD & $30^{*}$ & 9 & 22 & 15 & 16 & 7 & & & & & $23.4^{*}$ & g* & $16^{*}$ & $12^{*}$ & $21^{*}$ \\
\hline Obesity & 29 & 23 & & & & & & & 40 & 34 & $40.9^{*}$ & & 2 & 29 & \\
\hline DJD & 29 & & 22 & 20 & & & & & 55 & 37 & $58.6^{*}$ & & & & \\
\hline Diabetes mellitus & 22 & 54 & 8 & 7 & 19 & 11 & $7^{*}$ & 5 & 16 & 13 & $13.9^{*}$ & $14^{*}$ & $25^{*}$ & 28 & \\
\hline GERD & 18 & & & & & & & & & & & & & & \\
\hline PAD & 17 & 53 & & & & & $6^{*}$ & 2 & & & & & $18^{*}$ & $17^{*}$ & \\
\hline Depression & 17 & 16 & 17 & 13 & 7 & 5 & $g^{*}$ & 4 & 21 & 13 & $42.5^{*}$ & & & $15^{*}$ & \\
\hline CRF & 17 & 22 & 2 & 1 & & & & & 16 & 11 & & & $26^{*}$ & $16^{*}$ & $6^{*}$ \\
\hline CHF & $16^{*}$ & & 14 & 3 & 8 & 2 & & & 12 & & $15.1^{*}$ & $15^{*}$ & & $33^{*}$ & \\
\hline Underweight & 15 & 14 & & & & & & & & & & & & & \\
\hline Substance abuse & 14 & & & & & & & & & & & & & & \\
\hline Atrial fibrillation & $13^{*}$ & & & & & & & & & & & & $2^{*}$ & $21^{*}$ & \\
\hline Anxiety & $12^{*}$ & 21 & & & & & & & 9 & 4 & & & & 18 & \\
\hline $\begin{array}{l}\text { Erectile } \\
\text { dysfunction }\end{array}$ & 12 & & & & & & & & & & & & & & \\
\hline $\begin{array}{l}\text { Gastric/duodenal } \\
\text { ulcer }\end{array}$ & $12^{*}$ & & 32 & 17 & & & $7^{*}$ & 2 & & & & & $5^{*}$ & 10 & \\
\hline CVA & 11 & & 4 & 4 & & & $3^{*}$ & 4 & 9 & 5 & & & & $11^{*}$ & \\
\hline $\begin{array}{r}\text { Pulmonary } \\
H T N+C P\end{array}$ & $10^{*}$ & & & & & & & & & & & & $4^{*}$ & & $60^{*}$ \\
\hline OSA & 10 & & & & & & & & & & & & 2 & & \\
\hline
\end{tabular}


TABLE 2. Summary of COPD comorbidities studies describing the setting, methodology of disease ascertainment, number of subjects, prevalence, and impact on important outcomes (Continued)

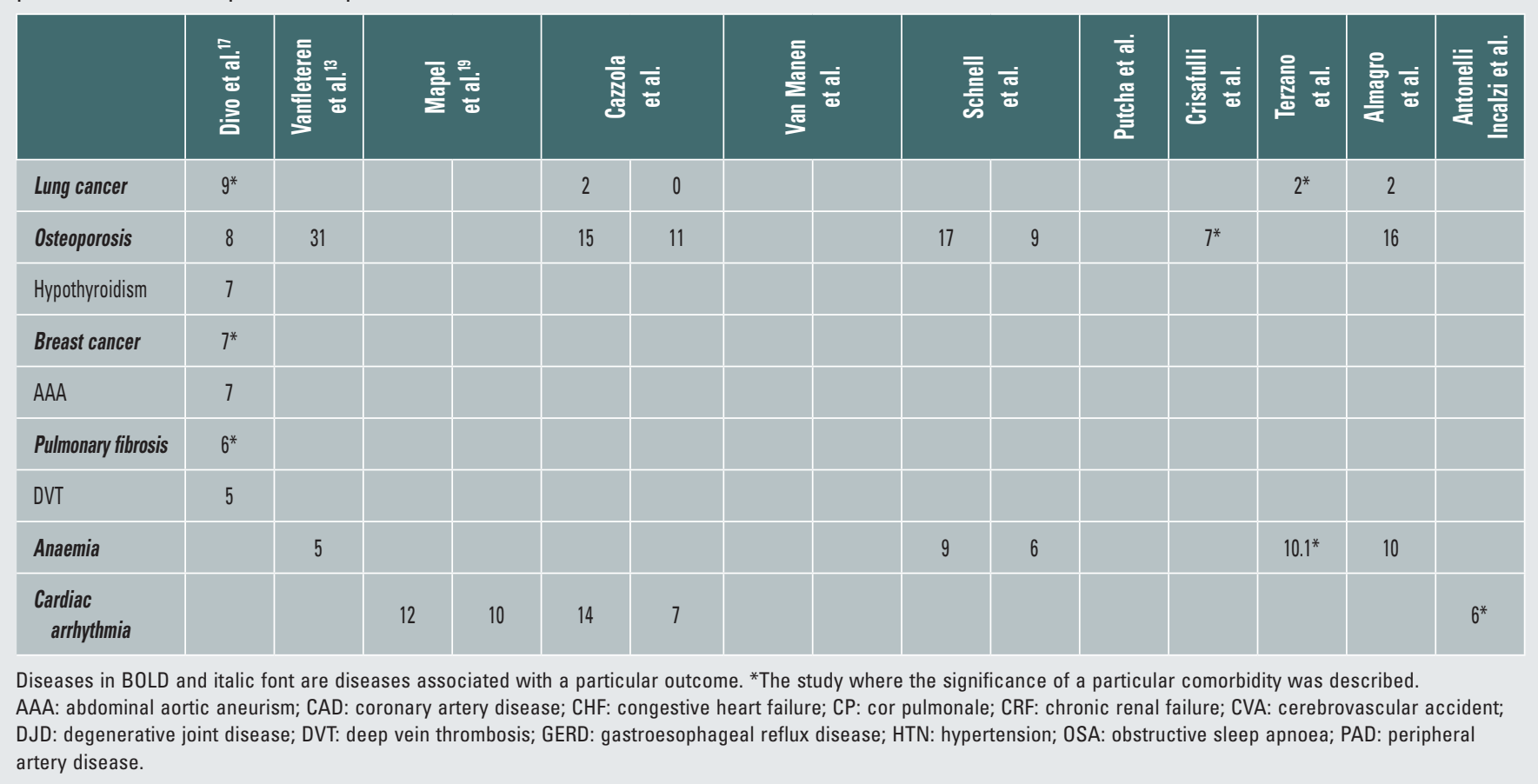

can be analysed using network theory. The application of network theory has helped to demonstrate that these complex systems have similar governing principles. These principles allow us to intuitively discover patterns, isolate structure singularities, and generate new hypotheses, and a full revision of those principles and examples were recently published in this journal ${ }^{28}$.

This methodology has been applied in the study of COPD and its comorbidities ${ }^{15,29-31}$, and we will highlight some of our own results. By constructing a network that includes COPD clinical characteristics and comorbidities from a cohort of 2,000 patients and compared to a non-COPD control group, we found: first, that the network of COPD-related comorbidities is denser than those without COPD (Fig. 5). The significance of this finding relates to the known fact that the number and prevalence of comorbidities is higher in COPD, but similar to social networks, in order to have more "followers" your network has to be popular and/or created with enough time in order to allow "followers" to join the network. It is also possible that the degenerative process leading to the development of chronic diseases starts at an early stage compared to those without COPD (and are smokers) ${ }^{32}$.

Second, by applying unbiased clustering algorithms, there are highly interconnected diseases that aggregate around patient's characteristics, revealing distinct clusters or modules. In figure 6 we show an example of a sub-network or module from the COPD Comorbidity Network (Fig. 5, right side). In this module, we observe that several significantly correlated diseases are clustered around demographic 


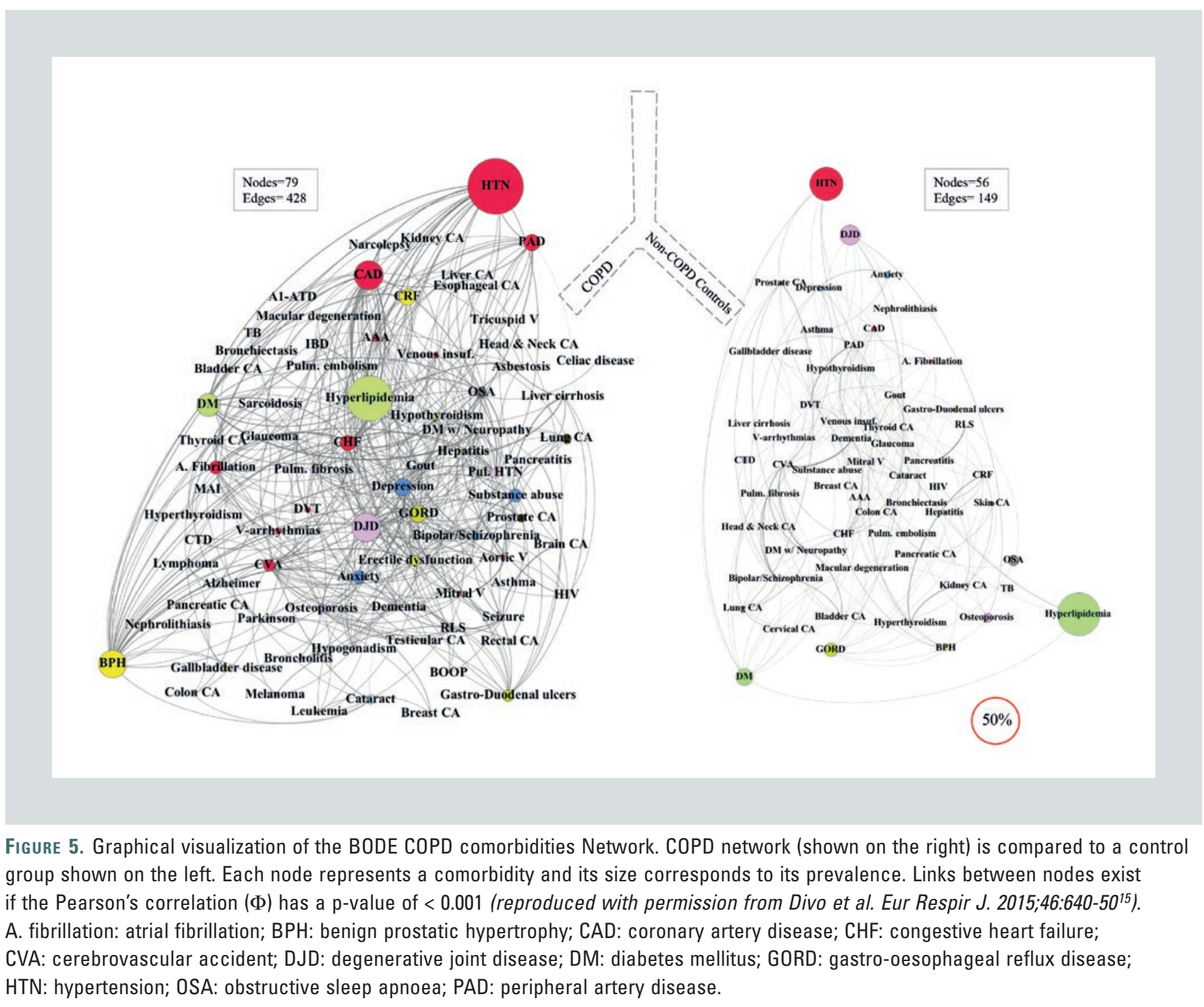

FIgURE 5. Graphical visualization of the BODE COPD comorbidities Network. COPD network (shown on the right) is compared to a control group shown on the left. Each node represents a comorbidity and its size corresponds to its prevalence. Links between nodes exist if the Pearson's correlation $(\Phi)$ has a p-value of < 0.001 (reproduced with permission from Divo et al. Eur Respir J. 2015;46:640-5015). A. fibrillation: atrial fibrillation; BPH: benign prostatic hypertrophy; CAD: coronary artery disease; CHF: congestive heart failure HTN: hypertension; OSA: obstructive sleep apnoea; PAD: peripheral artery disease.

and clinical characteristics. In figure 6 we see that COPD patients who are younger $(<55$ years old), underweight (body mass index $\left.<21 \mathrm{~kg} / \mathrm{m}^{2}\right)$, and current smokers are more likely to have comorbidities (HIV, hepatitis, pancreatitis, cirrhosis, cancers), to be associated with mental illness, and to engage in high-risk behaviour (anxiety, depression, and substance abuse).

Third, the pattern and distribution of connections between diseases (known as edge distribution) suggest a scale-free architecture of the network (long-tailed distribution where a few nodes have most of the links) and therefore we could identify certain diseases as representing highly connected hubs. Those hubs are illustrated in figure 7 and represent those highly connected comorbidities that hold $72 \%$ of the connections in the COPD comorbidity network, resembling the behaviour of large airports hubs like JFK in New York or Heathrow in London. Interestingly, these hubs are also known to be associated with important 


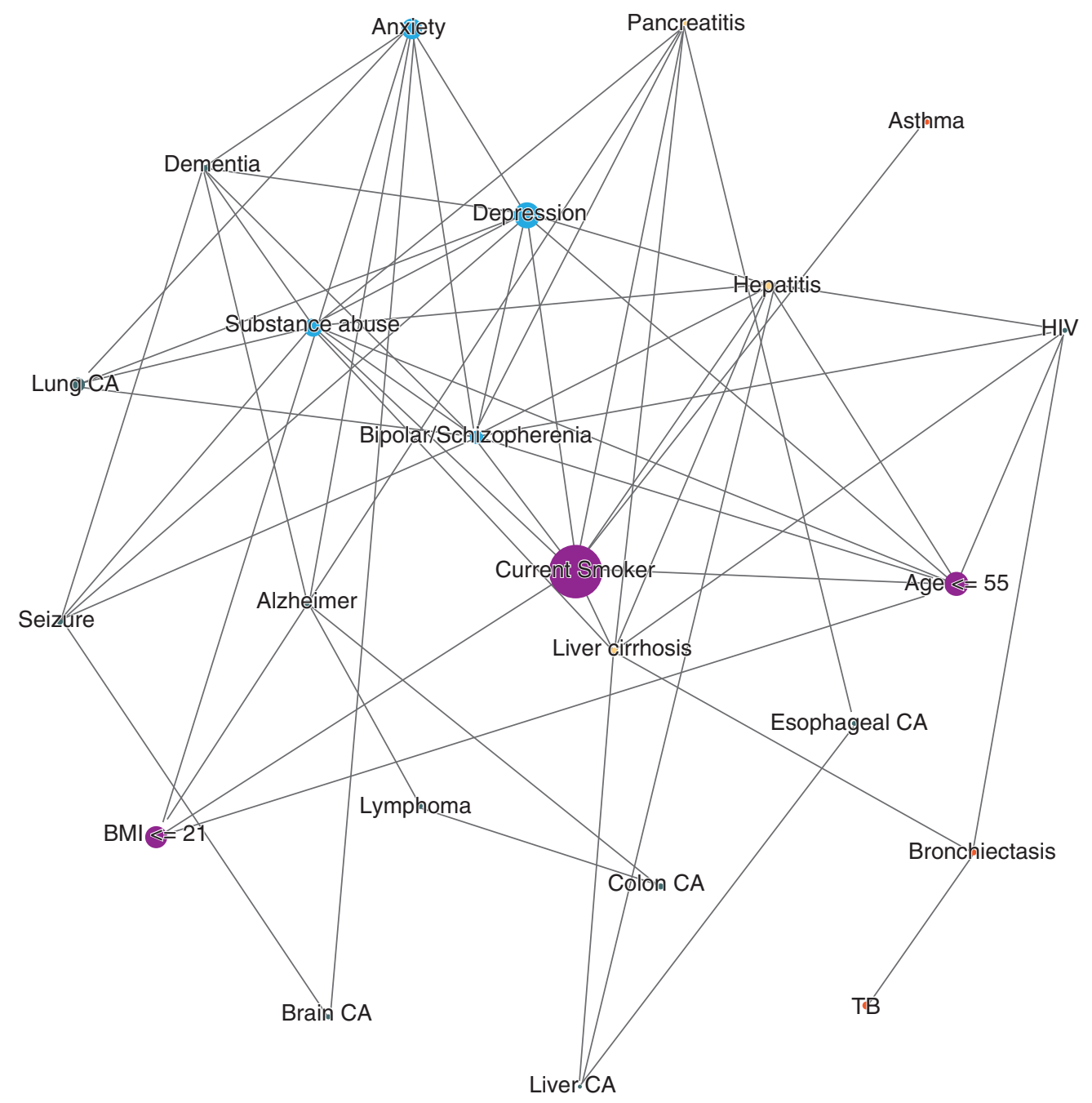

FiguRE 6. The psychiatric-behavioural Cluster extracted from the Comorbidity network (Fig. 5), using the modularity algorithm of network theory. The module contains correlated comorbidities and clinical characteristics tightly connected. The size of the nodes represents the prevalence and the links represent statistically significant correlations between connected nodes (reproduced with permission from Divo et al. Eur Respir J. 2015;46:640-5015).

BMI: Body mass Index CA: Cancer, TB: Tuberculosis.

patient-related outcomes like hospitalization ${ }^{33}$, quality of life ${ }^{34}$, and mortality ${ }^{35}$ as we previously presented in table 2 .

The identification of those hubs provides an interesting opportunity to generate new research hypotheses for intervention or for the identification of intermediary pathways that result in particular COPD phenotypes and comorbidity clusters ${ }^{30,36}$. We could conceive exploring what is known as network perturbation experiments, where we might 


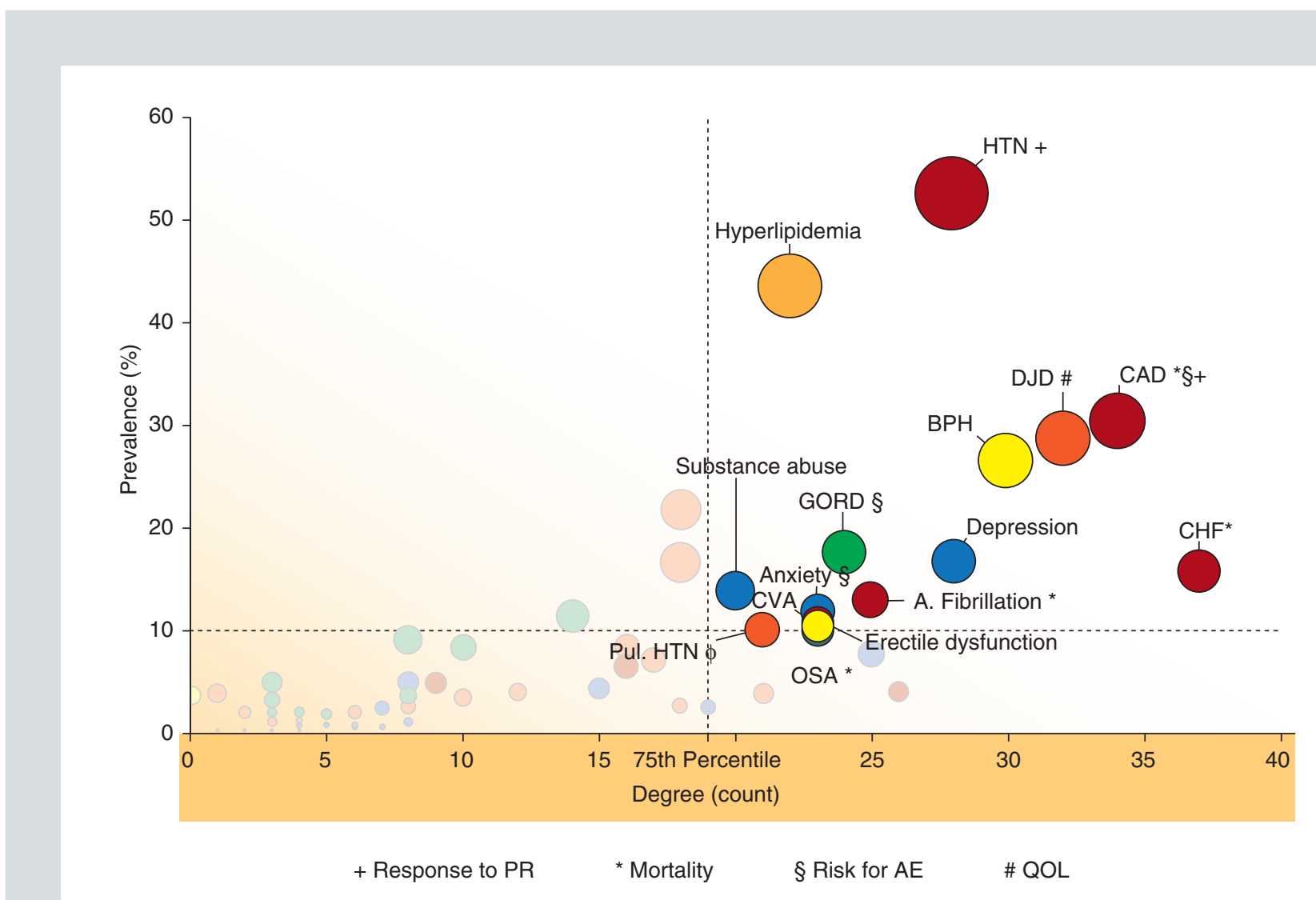

Figure 7. The relationship between prevalence and degree in the BODE comorbidity study. Disease with an ${ }^{*}, \#,+, \S$, represent disease that directly impact meaningful clinical outcomes (reproduced with permission from Divo et al. Chronic Obstructive Pulmonary Disease Comorbidities Network. Eur Respir J. 2015;46:640-50).

A. fibrillation: atrial fibrillation; AE: adverse events; $\mathrm{BPH}$ : benign prostatic hypertrophy; CAD: coronary artery disease; CHF: congestive heart failure; CVA: cerebrovascular accident; DJD: degenerative joint disease; DM: diabetes mellitus; GORD: gastro-oesophageal reflux disease; HTN: hypertension; OSA: obstructive sleep apnoea; PAD: peripheral artery disease; PR: pulmonary rehabilitation; 00L: quality of life.

therapeutically intervene one of those hubs and evaluate the response of the system. Perhaps, the benefits of targeting comorbidity hubs are greater than that of targeting single disease entities. As with air travel, delays (the perturbation) in large hubs have a greater effect on air traffic than delays in smaller airports.

For example, the use of CPAP (Continuous Positive Airway Pressure) has been shown to decrease mortality in patients with overlap COPD and obstructive sleep apnoea. Similarly, beta receptor blockers and angiotensin-converting enzyme inhibitor therapy may reduce mortality in patients with both COPD and cardiovascular disease ${ }^{37-39}$.

\section{WHERE DO WE GO FROM HERE?}

As we validate comorbidity clusters and their common pathways with particular COPD phenotypes and endotypes, we could realize the potential of personalized screening for 
at-risk populations by integrating the genetic, biologic, socioeconomic, and environmental risk with the use of bioinformatics and development of screening "algorithms". Those same algorithms that make the GOOGLE ${ }^{\circledR}$ search engine so valuable should be translated to the ever-growing use of electronic health records (EHR). The integration of data that we routinely collect in EHR has the potential for a more personalized approach to health if we interconnect, in a meaningful way, the different layers that influence health: social, environmental, occupational, genetic, and epigenetic exposures. In relation to COPD and tobaccorelated diseases, we could potentially identify the youngsters at risk for addiction to this and other substances to attempt to prevent the development of comorbidities as the person ages. This is probably the Holy Grail of personalized medicine. The current time is ripe as informatics has the potential to help disentangle complex data, a task that seemed impossible just a decade ago.

In summary, due to aging and a decrease in communicable diseases, humans are now reaching an older age and developing interactive multimorbidities. In fact, humans are complex systems and to better study the interaction of their genetic make-up with the environment, the application of system network analysis seems well suited to better understand this interaction as well as that of COPD with other chronic diseases.

\section{REFERENCES}

1. Affairs DOEAS, Population. United Nations, Department of Economic and Social Affairs, Population Division (2013). World Population Prospects: The 2012 Revision, Highlights and Advance Tables. Working Paper No. ESA/P/ WP.228. 2013
2. Kanapathipillai R, Henao Restrepo AM, Fast P et al. Ebola Vaccine - An Urgent International Priority. N Engl J Med. 2014;371:2249-51.

3. Kouznetsova J, Sun W, Martínez-Romero C et al. Identification of 53 compounds that block Ebola virus-like particle entry via a repurposing screen of approved drugs. Emerg Microbes Infect. 2014;3:e84.

4. Lee JE, Fusco ML, Hessell AJ et al. Structure of the Ebola virus glycoprotein bound to an antibody from a human survivor. Nature. 2008;454:177-82.

5. Barnett K, Mercer SW, Norbury M et al. Epidemiology of multimorbidity and implications for health care, research, and medical education: a crosssectional study. Lancet. 2012;380:37-43.

6. Prados-Torres A, Poblador-Plou B, Calderon-Larranaga A et al. Multimorbidity patterns in primary care: Interactions among chronic diseases using factor analysis. PLoS One. 2012;7.e32190.

7. Wang H, Dwyer-Lindgren L, Lofgren KT et al. Age-specific and sex-specific mortality in 187 countries, 1970-2010: a systematic analysis for the Global Burden of Disease Study 2010. Lancet. 2012;380:2071-94.

8. Hidalgo CA, Blumm N, Barabási A-L et al. A Dynamic Network Approach for the Study of Human Phenotypes. PLoS Comput Biol. 2009;5:e1000353.

9. McGarvey LP, John M, Anderson JA et al. Ascertainment of cause-specific mortality in COPD: operations of the TORCH Clinical Endpoint Committee. Thorax. 2007;62:411-5.

10. PAUWELS RA, Buist AS, Calverley PMA et al. Global Strategy for the Diagnosis, Management, and Prevention of Chronic Obstructive Pulmonary Disease. Am J Respir Crit Care Med. 2001;163:1256-76.

11. Vestbo J, Hurd SS, Agustí AG et al. Global Strategy for the Diagnosis, Management, and Prevention of Chronic Obstructive Pulmonary Disease. Am J Respir Crit Care Med. 2013;187:347-65.

12. Burgel PR, Paillasseur JL, Caillaud D et al. Clinical COPD phenotypes: a novel approach using principal component and cluster analyses. Eur Respir J. 2010;36:531-9.

13. Vanfleteren LE, Spruit MA, Groenen M et al. Clusters of comorbidities based on validated objective measurements and systemic inflammation in patients with chronic obstructive pulmonary disease. Am J Respir Crit Care Med. 2013;187:728-35.

14. Divo MJ, Cabrera C, Casanova C. Comorbidity distribution, clinical expression and survival in COPD patients with different body mass index. J COPD Foundation. 2014;1:229-38.

15. Divo MJ, Casanova C, Marin JM et al. Chronic obstructive pulmonary disease comorbidities network. Eur Respir J. 2015;46:640-50.

16. Garcia-Aymerich J, Gómez FP, Benet M et al. Identification and prospective validation of clinically relevant chronic obstructive pulmonary disease (COPD) subtypes. Thorax. 2011;66:430-7.

17. van Manen JG, Bindels PJ, IJzermans CJ et al. Prevalence of comorbidity in patients with a chronic airway obstruction and controls over the age of 40 . J Clin Epidemiol. 2001;54:287-93.

18. Mapel DW, Hurley JS, Frost FJ et al. Health care utilization in chronic obstructive pulmonary disease. A case-control study in a health maintenance organization. Arch Intern Med. 2000;160:2653-8.

19. Cazzola M, Bettoncelli G, Sessa E et al. Prevalence of Comorbidities in Patients with Chronic Obstructive Pulmonary Disease. Respiration. 2010; 80:112-9.

20. Schnell K, Weiss CO, Lee T et al. The prevalence of clinically-relevant comorbid conditions in patients with physician-diagnosed COPD: a cross-sectional study using data from NHANES 1999-2008. BMC Pulm Med. 2012;12:26.

21. Putcha N, Han MK, Martinez $\mathrm{CH}$ et al. Comorbidities of COPD have a major impact on clinical outcomes, particularly in African Americans. Chronic Obstr Pulm Dis. 2014;1:105-14.

22. Crisafulli E, Gorgone P, Vagaggini B et al. Efficacy of standard rehabilitation in COPD outpatients with comorbidities. Eur Respir J. 2010;36:1042-8.

23. Terzano C, Conti V, Di Stefano F et al. Comorbidity, Hospitalization, and Mortality in COPD: Results from a Longitudinal Study. Lung. 2010;188:321-9.

24. Almagro P, Cabrera FJ, Diez J et al. Comorbidities and short-term prognosis in patients hospitalized for acute exacerbation of COPD: the EPOC en Servicios de medicina interna (ESMI) study. Chest. 2012;142:1126-33. 
25. Incalzi RA, Fuso L, De Rosa M et al. Co-morbidity contributes to predict mortality of patients with chronic obstructive pulmonary disease. Eur Respir J. 1997;10:2794-800.

26. Menche J, Sharma A, Cho MH et al. A diVIsive Shuffling Approach (VIStA) for gene expression analysis to identify subtypes in Chronic Obstructive Pulmonary Disease. BMC Syst Biol. 2014;8(Suppl 2):S8.

27. Rennard SI, Locantore N, Delafont B et al. Identification of five chronic obstructive pulmonary disease subgroups with different prognoses in the ECLIPSE cohort using cluster analysis. Ann Am Thorac Soc. 2015;12: 303-12.

28. Agusti A, Lopez-Giraldo A, Cruz T, Faner R. Relevance of systems biology to respiratory medicine. BRN Rev. 2016;2:1-13.

29. Diez D, Agustí A, Wheelock CE. Network Analysis in the Investigation of Chronic Respiratory Diseases: from Basics to Application. Am J Respir Crit Care Med. 2014;190:981-8.

30. Grosdidier S, Ferrer A, Faner R et al. Network medicine analysis of COPD multimorbidities. Respir Res. 2014;15:111.

31. Faner R, Gutiérrez-Sacristán A, Castro-Acosta A et al. Molecular and clinical diseasome of comorbidities in exacerbated COPD patients. Eur Respir J. 2015;46:1001-10.

32. Ito K, Barnes PJ. COPD as a disease of accelerated lung aging. Chest. 2009;135:173-80.
33. Xu W, Collet JP, Shapiro S et al. Independent effect of depression and anxiety on chronic obstructive pulmonary disease exacerbations and hospitalizations. Am J Respir Crit Care Med. 2008;178:913-20.

34. Frei A, Muggensturm P, Putcha N et al. Five comorbidities reflected the health status in patients with chronic obstructive pulmonary disease: the newly developed COMCOLD index. J Clin Epidemiol. 2014;67:904-11.

35. Divo MJ, Cote C, de Torres JP et al. Comorbidities and risk of mortality in patients with chronic obstructive pulmonary disease. Am J Respir Crit Care Med. 2012;186:155-61.

36. Faner R, Cruz T, Lopez-Giraldo A et al. Network medicine, multimorbidity and the lung in the elderly. Eur Respir J. 2014;44:775-88.

37. Marin JM, Soriano JB, Carrizo SJ et al. Outcomes in patients with chronic obstructive pulmonary disease and obstructive sleep apnea: The Overlap Syndrome. Am J Respir Crit Care Med. 2010;182:325-31.

38. Etminan M, Etminan M, Jafari S et al. Beta-blocker use and COPD mortality: a systematic review and meta-analysis. BMC Pulm Med. 2012;12:48.

39. Mancini GB, Etminan M, Zhang B et al. Reduction of morbidity and mortality by statins, angiotensin-converting enzyme inhibitors, and angiotensin receptor blockers in patients with chronic obstructive pulmonary disease. J Am Coll Cardiol. 2006;47:2554-60.

40. Barabási A-L, Gulbahce N, Loscalzo J. Network medicine: a network-based approach to human disease. Nat Rev Genet. 2011;12:56-68. 